\title{
DETERMINATION OF CONTACT PRESSURE AT PNEUMATIC SEAL/ROD INTERFACE FROM RADIAL FORCE MEASUREMENT
}

\author{
GUIDO BELFORTE ${ }^{1}$, MARCELLO CONTE ${ }^{2}$, ANDREA MANUELLO ${ }^{3}$, LUIGI MAZZA ${ }^{1}$, \\ TERENZIANO RAPARELLI ${ }^{1} \&$ CARMEN VISCONTE ${ }^{1}$ \\ ${ }^{1}$ Department of Mechanical and Aerospace Engineering, Politecnico di Torino, Italy \\ ${ }^{2}$ Anton Paar Tritec SA, Switzerland \\ ${ }^{3}$ Department of Mechanical, Chemical and Material Engineering, Università di Cagliari, Italy
}

\begin{abstract}
This work relates to the experimental evaluation of contact pressure at the interface between an elastomeric rod seal for pneumatic cylinders and its metallic counterpart, without interposing any intrusive measuring device. Results were obtained using a suitable test bench able to detect the radial force exerted by the rod seal, displaced at constant velocity on a sensorized portion of a cylinder rod over time. Pressure load was applied on the seal to reproduce actual working conditions. A data postprocessing methodology was developed for an indirect evaluation of contact pressure, starting from the experimental data set of the radial force exerted by the seal on the rod. At first, the measured radial force signal was filtered and properly fitted, obtaining a differentiable function; then, contact pressure distribution was computed as a function of the radial force time derivative, seal velocity and rod diameter. The experimental test bench and the computational methodology described can be applied to pneumatic rod seals with geometries and materials other than that described in this study.

Keywords: contact pressure, radial force measurement, seals, pneumatics.
\end{abstract}

\section{INTRODUCTION}

The performance analysis of an elastomeric sealing device is a particularly complex and time-consuming goal not only because of the variety of used materials, shapes and dimensions, but also because of the relevant influence of many operating parameters, such as applied loads, working pressure, lubricating conditions, temperature, and velocity. In particular, the study of the contact characteristics at the rubber-metal interface is of great importance because both the sealing capability and the strength of the friction force depend on the contact pressure.

Much research has been addressed at the evaluation of contact pressure between a seal and its counterpart. Bignardi et al. [1] processed experimental data obtained on a pneumatic lip seal by a photo elastic reflection technique to compute analytically the contact pressure distribution at the seal-rod interface. Yang and Salant [2] developed an analytical procedure involving contact pressure calculation to evaluate the performance of hydraulic rod seals placed in a tandem arrangement. Pinedo et al. [3] built an analytical tri-dimensional eccentricity model of a rod lip seal extrapolating contact pressure distribution from finite elements simulations and experimental measurements. Hermann and Dabisch evaluated numerically the contact pressure distribution between a pneumatic rod seal and its countersurface [4]. Many research works reported the use of pressure sensitive films to determine experimentally contact pressure distribution over a friction couple, in various research fields. As an example, Misiewicz et al. [5] employed a piezoelectric pressure mapping system to measure contact area and contact pressure between an agriculture tyre and the soil, obtaining accurate distribution at the contact surface. Lee et al. [6] employed film sensors for measuring contact area and maximum contact pressure on a lip seal for hydraulic rotating shafts, under different interference fits. Pressure sensitive films were also 
employed for static measurements of local contact pressure in pneumatic applications by Belforte et al. [7]-[9] and by Manuello Bertetto et al. [10]; it was seen that these sensors were effective in firstly estimating the contact characteristics (contact pressure and contact area). Nevertheless, a limit of film sensors is due to their non-negligible thickness and to the difficulty of positioning them between the two parts in contact [11]. Some other authors reported non-intrusive experimental methods. An ultrasonic technique, which has the advantage of determining actual contact conditions, was used by Marshall et al. [12] in a bolted connection. Debler et al. [13] developed a test bench equipped with a specifically designed force sensor to measure contact pressure at a seal-rod interface indirectly. The radial force exerted by a seal mounted on a sensor-equipped rod was recorded over time and contact pressure was obtained by differentiating this signal.

This work relates with the experimental evaluation of contact pressure at the interface between an elastomeric rod seal for pneumatic cylinders and its metal counterpart. Results were obtained using a suitable test bench able to detect the radial force exerted by the rod seal displaced at constant velocity on a sensorized portion of a cylinder rod over time. With respect to Debler et al. [13], the measurement technique does not require the use of a specifically designed sensor; in fact, common uniaxial load cells were used to measure the radial force. Furthermore, the developed test bench permits to apply a pressure load on the seal, to reproduce actual working conditions. A data postprocessing methodology was developed for an indirect evaluation of contact pressure starting from the experimental data set of the radial force exerted by the seal on the rod. At first, the measured radial force signal was filtered and properly fitted obtaining a differentiable function; then, contact pressure distribution was computed as a function of the radial force time derivative, seal velocity and rod diameter.

\section{EXPERIMENTAL METHODOLOGY}

In order to contribute to a better understanding of the adopted measuring set-up, Fig. 1 shows a scheme of the seal, assembled on the test bench. The rod seal, which is loaded by compressed air pressure on the left side, is pulled at constant velocity over a fixed cylinder rod (1), whose final portion is split into two sectors: the first one is an extension of the rod body ( 1 '), the second one (2) is free to move in y-direction and is connected to a force sensor. This sensor, which is not shown in the figure, allows measuring the overall radial force oriented in the negative y-direction and exerted by the seal under test on the rod portion (2). To this aim, a gap (3) is provided between the rod sectors, which permits a relative frictionless motion. During the test, the seal initially slides over the full rod (Fig. 1a); then, it gradually engages the sensorized portion of the rod (Fig. 1b); finally, it completely covers the sensorized rod tip (Fig. 1c). While the contact area between the seal and the sensorized rod tip increases, the sensor detects an increasing radial force until a maximum value is reached.

Since the seal motion occurs at constant velocity, the relationship between the radial force trend line over time and the contact pressure distribution over the contact surface can be identified as follows.

With reference to Fig. 2, representing a portion of the sensor-equipped rod (diameter $d$ ), the measured radial force $F$ is the resultant of the contact pressure $p$ distribution over the semi-rod circular surface.

The balance equation (1) along the $y$ direction is:

$$
d F(z)=d z \cdot 2 \cdot \int_{0}^{\frac{\pi}{2}} p(z) \cdot \frac{d}{2} \cdot \sin \alpha \cdot d \alpha
$$




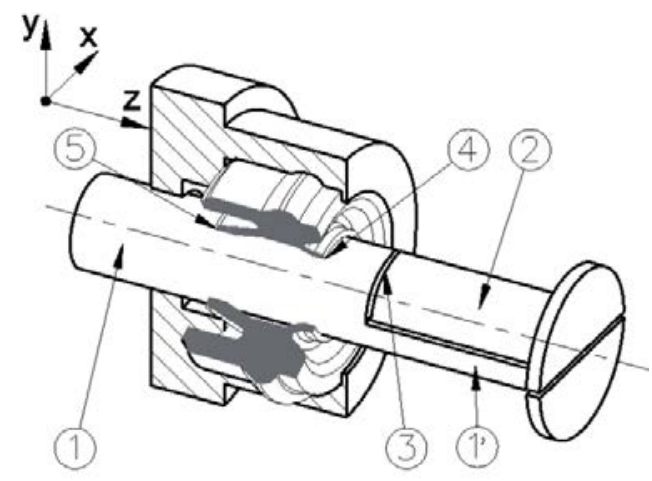

a)

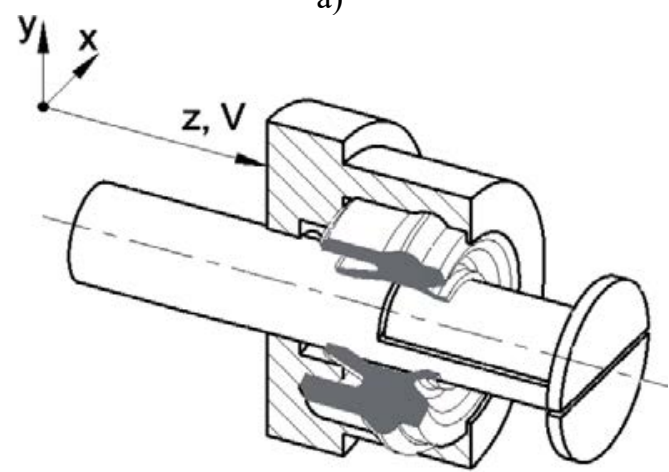

b)

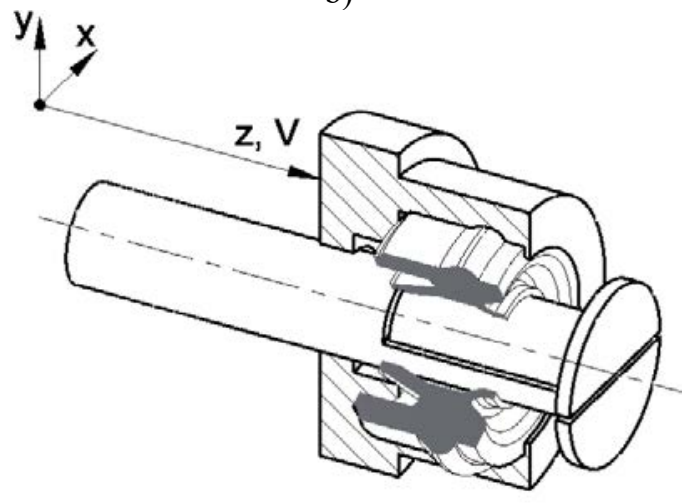

c)

Figure 1: Dynamic measurement of the seal/rod radial force.

By integrating, it will be obtained:

$$
d F(z)=d z \cdot p(z) \cdot d
$$

Dividing both terms of eqn (2) by time dt, $p(z)$ can be obtained as:

$$
p(z)=\frac{d F(z)}{d t} \cdot \frac{d t}{d z} \cdot \frac{1}{d}=\frac{d F(z)}{d t} \cdot \frac{1}{V \cdot d},
$$

where $V$ is the constant sliding velocity of the seal along the $z$-direction. 


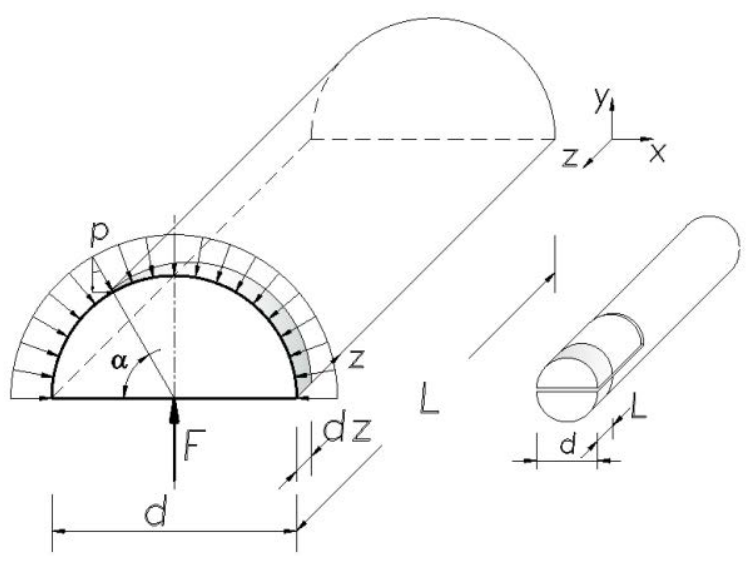

Figure 2: Sensor-equipped portion of the rod.

This measurement method reproduces actual working conditions without introducing hardly any additional deformations of the seal; this characteristic represents a great advantage with respect to other experimental methods, as those making use of pressure sensitive films.

\section{EXPERIMENTAL TESTS}

The dynamic measurement of the seal/rod radial force was carried out using a suitably designed test bench. A linear actuator, whose velocity can be controlled within a range of $0,5-300 \mathrm{~mm} / \mathrm{s}$, pulls the seal holder, which is supported by a pneumo-static journal bearing. This way, the seal displacement is performed without introducing additional friction forces. Furthermore, the test bench is equipped by pneumo-static pads both to ensure a precise relative positioning between the sensorized rod portion (2) and the rod (1), balancing any misaligning force or moment, and allowing low friction displacement of the rod portion (2) along the direction of radial force transmission (negative y-direction). The seal holder is designed so to permit pressurization of the sealing lip of the seal, simulating actual working conditions. More details on the test bench can be found in Belforte et al. [14].

An elastomeric double lip seal for a pneumatic cylinder with a $20-\mathrm{mm}$ rod diameter was chosen to perform experiments. Nevertheless, the experimental test bench and the computational methodology described are general and can be applied to pneumatic rod seals with geometries and materials other than that described in this study. Fig. 3 shows a cross-section of the seal, provided by a wiper lip (4) and a sealing lip (5).

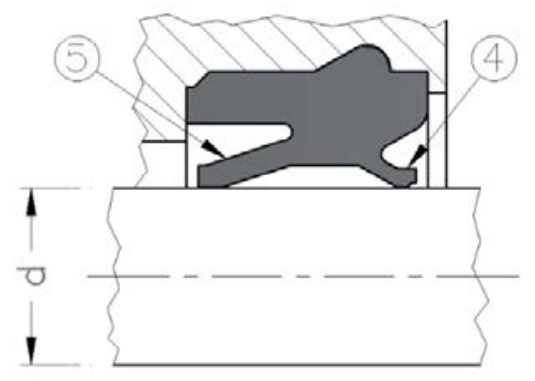

Figure 3: Cross-section of the seal under test. 
Tests were carried out pulling the seal holder at a constant velocity of $1 \mathrm{~mm} / \mathrm{s}$.

\subsection{Dynamic seal/rod radial force}

Fig. 4 shows an example of radial force trend over time, obtained applying a pressure load of 6 bar (gauge pressure) on the seal.

As shown, while the seal is sliding over the intact rod, the force detected by the sensor is equal to zero; then, when the wiper lip engages the sensorized portion of the rod, the radial force increases and subsequently remains almost stable. When also the sealing lip, loaded by air pressure, passes over the sensorized rod tip, an abrupt increase of force is detected. Later, the force signal falls down instead of remaining stable at a constant value. It is due to the fact that, when the sealing lip passes over the gap provided between the rod sectors (gap (3) in Fig. 1), the compressed air used to load the seal is quickly exhausted.

It can be noted that a certain noise and some spikes affect the signal given by the force sensor. According to the methodology previously described, this signal must be differentiated to obtain the seal/rod contact pressure distribution; therefore, the radial force signal was filtered and splitted in three parts, which were fitted by polynomial functions. There were some discontinuities among the fitted curves, involving few milliseconds, corresponding to the zones where the first derivative varied too much. Therefore, having calculated the first time derivative of each fitting function, the continuity among them was finally imposed.

Fig. 5 shows the three fitted curves, together with their first time derivative, connected one to the other.

\subsection{Contact pressure distribution}

Fig. 6 shows the contact pressure distribution between the double lip seal and the rod, as obtained applying eqn (3), i.e. dividing the time derivative signal by the product of the

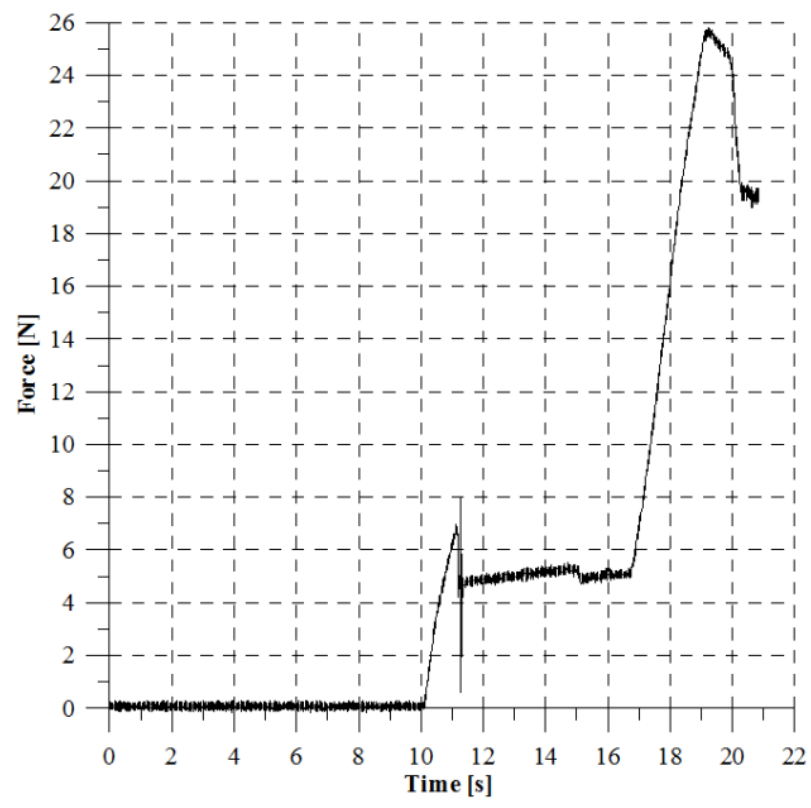

Figure 4: radial force trend over time. 


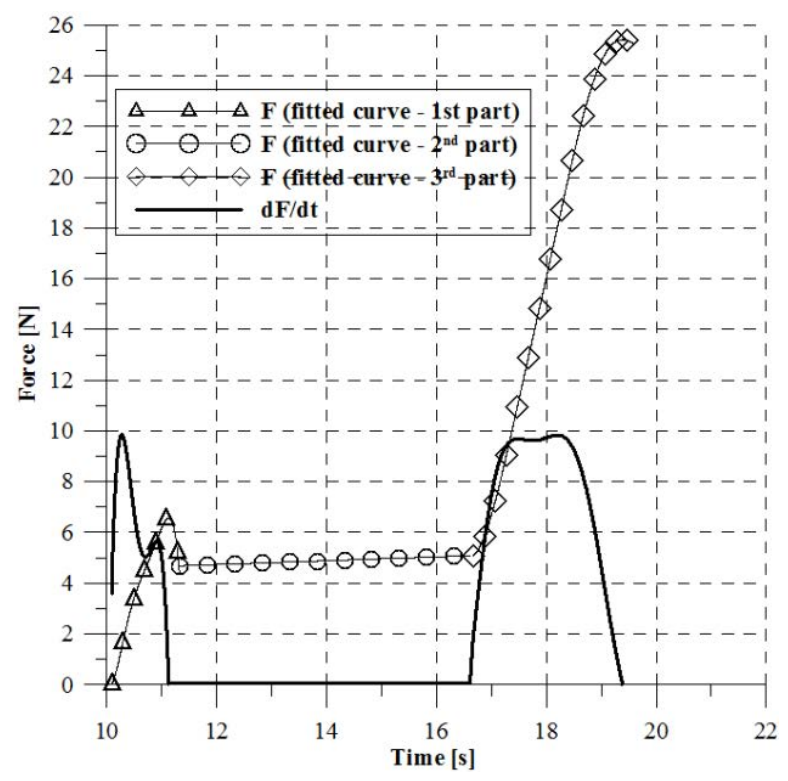

Figure 5: fitting and differentiation of experimental data.

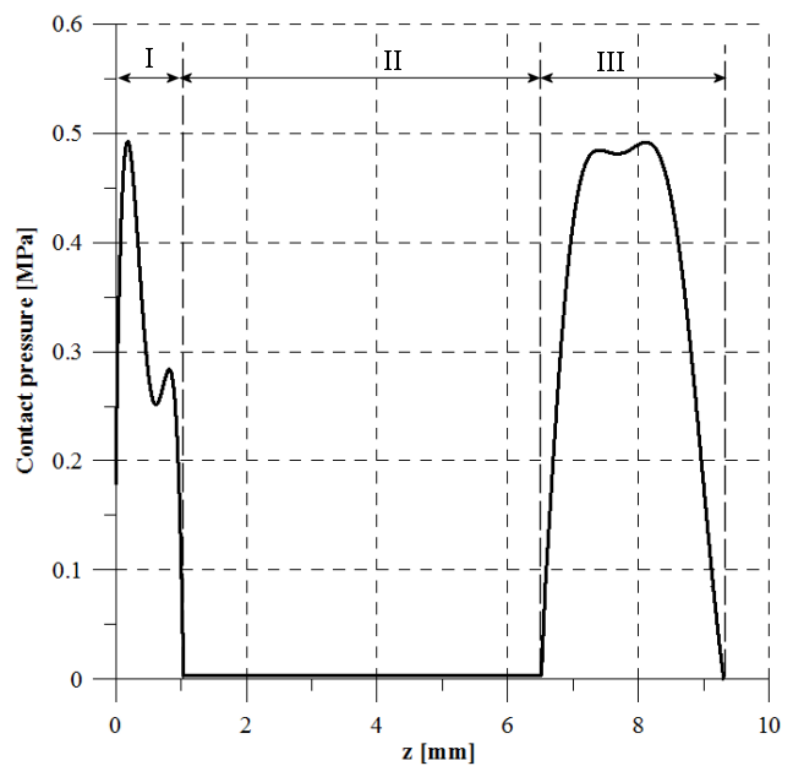

Figure 6: seal/rod contact pressure distribution.

sliding velocity $V$ and the rod diameter $d$. Since both $V$ and $d$ remains constant during the test, their product simply acts as a scale factor between the trend of force time derivative versus time and the trend of contact pressure distribution versus the $z$ coordinate, i.e. along 
the seal/rod contact surface. The relationship between the time and the $z$ coordinate is simply given by $z=V \cdot t$.

Results are comparable to those found by Hermann and Dabisch in [4] on a similar pneumatic rod seal.

With reference to Fig. 6, it must be remarked that Part I and Part III refer to the wiper lip/rod contact surface and to the sealing lip/rod contact surface, respectively. It can be noted that the radial force exerted by the sealing lip, higher than that exerted by the wiper lip because of the effect of the compressed air load, results in a contact pressure distribution characterized by the same level of that obtained on the wiper lip, but spread over a larger contact area.

\section{CONCLUSIONS}

Results of experimental tests aimed at the evaluation of contact pressure at the interface between an elastomeric rod seal for pneumatic cylinders and its metallic counterpart are presented. Tests were carried out using a suitable test bench able to detect the radial force exerted by the rod seal displaced at constant velocity on a sensorized portion of a cylinder rod over time. Pressure load was applied on the seal to reproduce actual working conditions. No intrusive measuring device was interposed between the tribological couple, which is the great advantage of the presented method. Experimental results, which gave the radial force trend over time, were postprocessed to indirectly calculate contact pressure distribution. In particular, the radial force signal was filtered and splitted in parts, which were fitted by polynomial functions. Finally, these functions were linked one to the other. This way, the radial force signal could be differentiated and contact pressure distribution was computed as a function of the radial force time derivative, seal velocity and rod diameter.

\section{REFERENCES}

[1] Bignardi, C., Manuello, A. \& Mazza, L., Photo elastic measurements and computation of the stress field and contact pressure in a pneumatic lip seal. Tribology International, 32(1), pp. 1-13, 1999.

[2] Yang, B. \& Salant, R.F., Numerical model of a tandem reciprocating hydraulic rod seal. Journal of Tribology, 130(3), pp. 032201.1 - 032201.7, 2008.

[3] Pinedo, B., Aguirrebeitia, J., Conte, M. \& Igartua, A., Tri-dimensional eccentricity model of a rod lip seal. Tribology International,78, pp. 68-74, 2014.

[4] Hermann, A. \& Dabisch, T., Influence on tribological behaviour of pneumatic actuators due to modifications of polymer compounds. Proceedings of the 15th ISC International Sealing Conference, pp. 265-280, 2008.

[5] Misiewicz, P.A., Richards, T.E., Blackburn, K., Brighton, J.L., Hann, M.J. \& Godwin, R.J., Techniques for estimating contact pressure resulting from loaded agriculture tyres. American Society of Agricultural and Biological Engineers Annual International Meeting 2008, 1, pp. 521-535, 2008.

[6] Lee, C.Y., Lin, C.S., Jian, R.Q. \& Wen C.Y., Simulation and experimentation of the contact width and pressure distribution of lip seals. Tribology International, 39(9), pp. 915-920, 2006.

[7] Belforte, G., Conte, M., Manuello, A., Mazza, L. \& Visconte, C., Experimental and numerical evaluation of contact pressure in pneumatic seals. Tribology International, 42(1), pp. 169-175, 2009. 
[8] Belforte, G., Conte, M. \& Mazza L., Study on pneumatic cylinder piston seals behaviour. 15th International Sealing Conference, Stuttgart, Germany, 7-8 October 2008.

[9] Belforte, G., Conte, M., Manuello, A., Mazza, L. \& Visconte, C., Contact pressure measurements in pneumatic seals by means of sensitive films. Sealing for Pollution Prevention and Control - 19th International Conference on Fluid Sealing, Poitiers, France, 2007.

[10] Manuello Bertetto, A., Mazza, L. \& Orrù, P.F., Contact pressure distribution in guide bearings for pneumatic actuators. Experimental Techniques, 39(2), pp. 46-54, 2015.

[11] Wu, J.Z., Herzog, W. \& Epstein M., Effect of inserting a pressensor film into articular joints on the actual contact mechanics. Journal of Biomechanical Engineering, 120, pp. 655-659, 1998.

[12] Marshall, M.B., Lewis, R. \& Dwyer-Joyce, R.S., Characterisation of contact pressure distribution in bolted joints. Strain, 42(6), pp. 31-43, 2006.

[13] Debler, C., Gronitzki, M. \& Poll, G., Investigation into the sealing contacts of reciprocating elastomeric seals-correlation of calculations with measurements and optical observations. 17th International Conference on Fluid Sealing, York, U.K., pp. 169-186, 2003.

[14] Belforte, G, Conte, M., Mazza, L., Raparelli, T. \& Visconte, C., Test rig for rod seals contact pressure measurement. WIT Transactions on Engineering Sciences, 66, WIT Press: Southampton and Boston, pp. 107-114, 2010. 